\title{
Denaturing Gradient Gel Electrophoresis Profiles of 16S rRNA-Defined Populations Inhabiting a Hot Spring Microbial Mat Community
}

\author{
M. J. FERRIS, ${ }^{1 *}$ G. MUYZER ${ }^{2}$ AND D. M. WARD ${ }^{1}$ \\ Department of Microbiology, Montana State University, Bozeman, Montana 59717, ${ }^{1}$ and Max-Planck Institute \\ for Marine Microbiology, D-28359 Bremen, Germany ${ }^{2}$
}

Received 14 July 1995/Accepted 4 November 1995

\begin{abstract}
Denaturing gradient gel electrophoresis (DGGE) of PCR-amplified 16S rRNA gene segments was used to profile microbial populations inhabiting different temperature regions in the microbial mat community of Octopus Spring, Yellowstone National Park. DGGE allowed a rapid evaluation of the distributions of amplifiable sequence types. Profiles were essentially identical within regions of the mat defined by one temperature range but varied between sites with different temperature ranges. Individual DGGE bands were sequenced, and the sequences were compared with those previously obtained from the mat by cloning and from cultivated Octopus Spring isolates. Two known cyanobacterial populations and one known green nonsulfur bacteriumlike population were detected by DGGE, as were many new cyanobacterial and green nonsulfur and green sulfur bacterium-like populations and a novel bacterial population of uncertain phylogenetic affiliation. The distributions of several cyanobacterial populations compared favorably with results obtained previously by oligonucleotide probe analyses and suggest that adaptation to temperature has occurred among cyanobacteria which are phylogenetically very similar.
\end{abstract}

A reliable method for determining the diversity of microbes in an environmental sample has long been sought by microbiologists. Microscopy and cultivation have limited usefulness, since diverse microorganisms can share similar, simple morphologies and cultivation limits detection to organisms that grow under the conditions provided in culture media. The development of techniques for the analysis of $16 \mathrm{~S}$ rRNA sequences in natural samples has greatly enhanced our ability to detect and identify bacteria in nature $(22,23)$. This has confirmed suspicions that only a small percentage of bacterial species have been isolated in culture $(3-5,10,11,14,29,32)$.

Recently, denaturing gradient gel electrophoresis (DGGE) analysis of $16 \mathrm{~S}$ rRNA gene segments has been used to profile complex microbial communities (17) and to infer the phylogenetic affiliation of the community members $(16,19)$. In this method, the PCR is used to amplify a region of the 16S rRNA gene or a functional gene, such as the (NiFe) hydrogenase gene (34), from mixed microbial populations. One of the PCR primers includes a GC-rich sequence (GC clamp) on its 5' end that imparts melting stability to the PCR products in a denaturing gradient gel. The resulting products, essentially all the same size, are separated into discrete bands during electrophoresis through an acrylamide gel that contains an increasing linear gradient of denaturants. Individual double-stranded DNA molecules denature along their length adjacent to the GC clamp according to their melting characteristics (i.e., sequences). This partial denaturation causes their migration to essentially halt at unique positions, forming discrete bands in the gel. Individual bands can be probed by Southern blotting $(1,17)$, or they can be excised and the nucleotide sequences can be determined $(16,19)$. Useful information can also be

\footnotetext{
* Corresponding author. Mailing address: Department of Microbiology, Montana State University, Bozeman, MT 59717. Phone: (406) 994-3412. Fax: (406) 994-4926. Electronic mail address: mjf@ward.os cs.montana.edu.
}

obtained through analyses of banding patterns alone, since each band may represent a different microbial population.

By studying the microbial mat community in Octopus Spring, a thermal pool in the Lower Geyser Basin of Yellowstone National Park, we hope to explain the discrepancy between bacteria detected by cultivation and those detected by $16 \mathrm{~S}$ rRNA analysis. We have used cultivation, 16S rRNA cloning and sequencing methods, and oligonucleotide probe analyses to reveal the extent to which cultivation and microscopy have concealed microbial diversity and to examine the environmental factors that influence population distributions within the microbial mat community $(9,27,30-33,35)$. Cultivation and $16 \mathrm{~S}$ rRNA-based cloning and sequencing methods have revealed seven cyanobacterial populations, two green nonsulfur bacterium-like populations, three green sulfur bacterium-like populations, and many other apparently nonphototrophic bacterial populations of various phylogenetic affiliations $(9,31,35)$. However, cloning and sequencing are very time-consuming, and oligonucleotide probing of dozens of individual populations to evaluate distributions with respect to environmental parameters is also an arduous task. The potential for DGGE to offer a more rapid and comprehensive means to simultaneously survey various bacterial populations prompted us to evaluate the technique in our system. Here, we examine spatial heterogeneity of microbial populations within and among regions of the mat defined by temperature. DGGE banding patterns were evaluated, and bacterial populations were identified by sequencing individual bands. We compare the DGGE results with the $16 \mathrm{~S}$ rRNA sequences obtained by direct cloning and cultivation and with previous oligonucleotide probe distribution analyses.

\section{MATERIALS AND METHODS}

Octopus Spring mat samples. Samples were collected on 17 August 1994 from the shoulder region of Octopus Spring, where the mat is covered by a few centimeters of water. Three sites differing with respect to their temperature profiles were located by placing a disk-shaped thermistor probe (ca. 1-cm diam- 
eter) on the mat surface and recording the temperature fluctuations for several minutes. Three separate samples were collected within $10 \mathrm{~cm}$ of the probe at each site. For each sample, approximately $5 \mathrm{ml}$ of mat material was obtained by scraping the upper 1 to $2 \mathrm{~mm}$ into sterile polypropylene tubes to which approximately $5 \mathrm{ml}$ of water from the site had been added. The samples were frozen on dry ice in the field and maintained at $-70^{\circ} \mathrm{C}$ until DNA was extracted.

DNA extraction. Each mat sample was homogenized in a sterile 40-ml tissue grinder until the suspension could be easily pipetted. Approximately $0.5 \mathrm{ml}$ of mat homogenate was used for DNA extraction. Cells were lysed in a MiniBeadbeater (Biospec Products, Bartlesville, Okla.) using sterile 0.1-mm-diameter zirconium beads according to the manufacturer's directions. DNA was obtained from the lysate by using standard phenol-chloroform extraction and ethanol precipitation procedures (15).

DGGE standards. A set of six Octopus Spring DGGE standards was generated by PCR amplification of DNA representative of known cyanobacterial and green nonsulfur bacterium 16S rRNA sequence types. Three of these markers (C9, $\mathrm{B} 10$, and $\mathrm{C} 1$ ) were generated from DNA extracted from cyanobacterial isolates which had been previously cultivated from a $50^{\circ} \mathrm{C}$ region of the mat in the shoulder area of Octopus Spring (9). The DNA extraction procedure for cell pellets was essentially as described for the mat samples. Two of these isolates (C9 and B10) are numerically relevant members of the Octopus Spring cyanobacterial community, since they were cultivated from extincting dilutions $(9,31)$. The $16 \mathrm{~S}$ rRNA sequence of isolate $\mathrm{B} 10$ is identical to that of sequence type $\mathrm{P}$, which was detected in a cloning library (the type $\mathrm{P}$ designation is used in all figures). The third cyanobacterial isolate (C1) has a 16S rRNA sequence that is identical to that of Synechococcus lividus Y-7c-s (9). Two other cyanobacterial DGGE markers were generated by PCR amplification of plasmid minipreps (15) of clones carrying the type A and B sequences (35). Another marker was prepared from Chloroflexus aurantiacus Y-400-fl cells as described for the cultivated cyanobacteria.

PCR primer design and rationale. We chose PCR primers in an attempt to maximize recovery of known Octopus Spring 16S rRNA genes of the domain Bacteria in a DNA region of the molecule for which we have the most sequence data and toward which previous studies had targeted specific oligonucleotide probes (27). One primer complements a region conserved among members of the domain Bacteria (Escherichia coli positions 1055 to 1070; primer sequence $5^{\prime}$-ATGGCTGTCGTCAGCT-3') (2). The other primer is based on a universally conserved region (E. coli positions 1392 to 1406; 5'-CGCCCGCCGCGC CCCGCGCCCGGCCCGCCGCCCCCGCCCCACGGGCGGTGTGTAC-3') (2) and incorporates a 40-base GC clamp. The specificity of this primer is imparted by the underlined region. Theoretically, these primers should amplify a 323-bp section of the 16S rRNA genes of members of the domain Bacteria, including the highly variable V9 region (12). A nested primer (5'-CCCGGGAACGTATT CACCGC-3') corresponding to E. coli positions 1368 to 1387 was used to obtain sequence data through this region.

PCR. PCRs were performed by using cloned Pfu DNA polymerase according to the instructions provided by the manufacturer (Stratagene, La Jolla, Calif.). Excess template DNA resulted in the production of nonspecific PCR products, which might appear as irrelevant bands in DGGE analysis. To eliminate this possibility, optimal concentrations of template were determined empirically by visual inspection of 10-fold serially diluted DNA samples on ethidium bromidestained agarose gels. One-microliter samples of the dilutions exhibiting the faintest template bands were PCR amplified. Of course, this results in possible loss of some target sequences, but predominant community members should still be represented. PCR products were examined on ethidium bromide-stained agarose gels, and the reaction mixtures yielding products of the expected size were used for DGGE analysis. The temperature cycle for the PCR was $1 \mathrm{~min}$ of denaturation at $94^{\circ} \mathrm{C}, 1 \mathrm{~min}$ of annealing (see below), and $3 \mathrm{~min}$ of primer extension at $72^{\circ} \mathrm{C}$. An initial denaturation step in which the temperature of the PCR mixture (without the polymerase) was raised to $94^{\circ} \mathrm{C}$ for $5 \mathrm{~min}$ and then lowered to $80^{\circ} \mathrm{C}$ for $1 \mathrm{~min}$, at which time the polymerase was added, was used. During an initial touchdown cycle, the annealing temperature was lowered from 53 to $43^{\circ} \mathrm{C}$ in intervals of $1^{\circ} \mathrm{C}$ per cycle; 20 additional annealing cycles were done at $43^{\circ} \mathrm{C}(7)$. The final primer extension was for $10 \mathrm{~min}$.

DGGE. All reagents and techniques were as previously described, except as noted $(1,18,20)$. Acrylamide gels $(6.5 \%)$ were prepared and run with $0.5 \times$ TAE buffer $(1 \times$ TAE is $0.04 \mathrm{M}$ Tris base, $0.02 \mathrm{M}$ sodium acetate, and 1.0 mM EDTA; $\mathrm{pH}$ adjusted to 7.4). A Hoeffer SE 600 gel electrophoresis unit was used with glass plates (16 by $18 \mathrm{~cm}), 1.5-\mathrm{mm}$ spacers, and 1-cm-wide loading wells. A 10-gallon (ca. 40-liter) aquarium served as the lower buffer chamber. DGGE gels contained a 35 to $80 \%$ gradient of urea and formamide (UF) solution increasing in the direction of electrophoresis. A $100 \%$ UF solution is defined as $40 \%$ ( vol/vol) formamide plus $7.0 \mathrm{M}$ urea $(1,20)$. DGGE was conducted at a constant voltage of $200 \mathrm{~V}$ at $60^{\circ} \mathrm{C}$ for $7 \mathrm{~h}$, after which the band positions had stabilized. The UF gradients and electrophoresis times were determined by perpendicular and time travel DGGE experiments $(1,17,20)$. Gels were stained with ethidium bromide $(0.5 \mu \mathrm{g} / \mathrm{ml})$ and photographed.

Sequencing of DGGE bands. Bands in DGGE gels were excised with a razor blade, and the small blocks of acrylamide containing the bands were placed in sterile $1.5-\mathrm{ml}$ bead-beater vials. The vials were filled to half their height with sterile 1-mm-diameter glass beads, and $0.5 \mathrm{ml}$ of sterile molecular biology-grade water (Sigma Chemical Co., St. Louis, Mo.) was added. The samples were then
A.
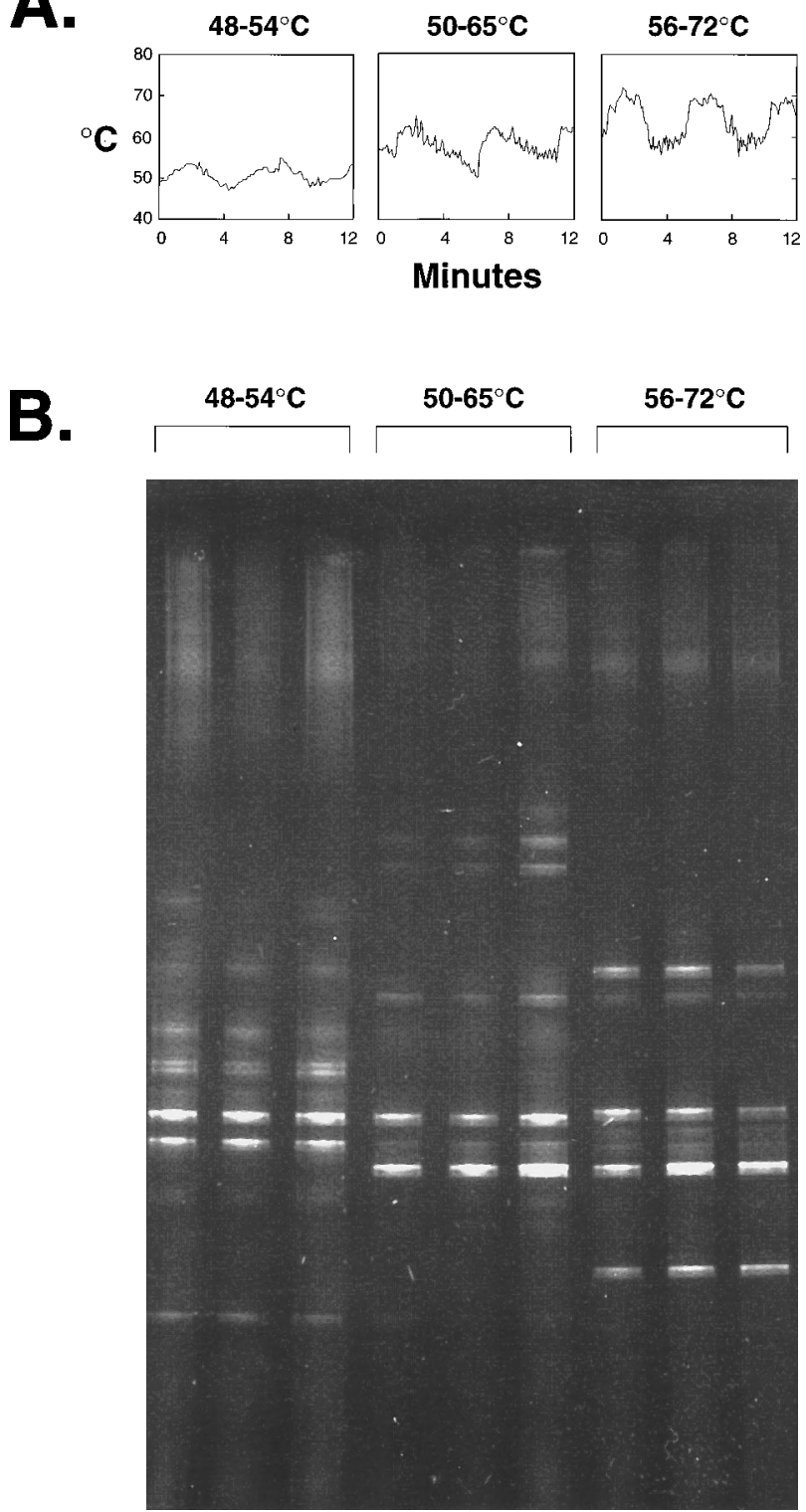

FIG. 1. (A) Temperature profiles of the three sampling sites examined by DGGE. (B) DGGE profiles of 16S rRNA-defined bacterial populations within triplicate samples at each site.

agitated in the Mini Beadbeater at the high setting for 3 min to disrupt the gel slice, placed at $4^{\circ} \mathrm{C}$ overnight to allow diffusion of DNA out of the gel fragments, and then centrifuged at low speed to pellet acrylamide fragments. Ten microliters of the supernatant was used as template DNA in a reamplification PCR using the primers and reaction conditions described above. Following amplification, the PCR products were rerun on DGGE gels to confirm their positions relative to the bands from which they were excised. The PCR products were then directly sequenced by using a Sequenase PCR product sequencing kit (United States Biochemical, Cleveland, Ohio) according to the manufacturer's directions. Sequence comparisons were performed after alignment to sequences in our Octopus Spring database and the Ribosomal Database Project as previously described (13).

Nucleotide sequence accession numbers. New sequence data have been submitted to GenBank, as follows: strain OS type A', U42374; strain OS type B', U42375; strain OS type E', U42419; strain OS type E'", U42420; strain OS type C', U42421; strain OS type Q, U42422.

\section{RESULTS}

Figure 1A shows the temperature profiles for the three re- 
A
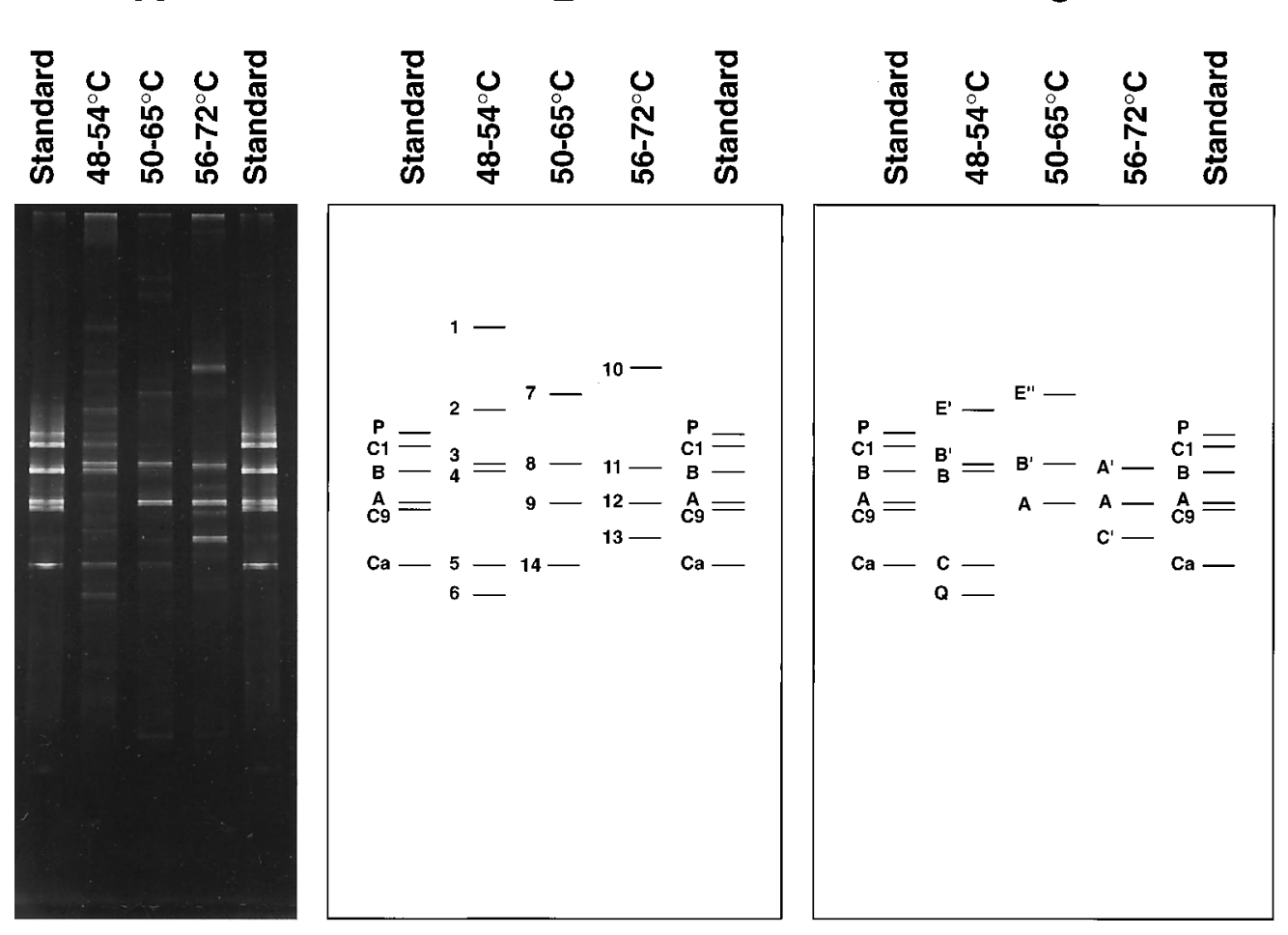

B

C

FIG. 2. (A) DGGE profiles of Octopus Spring mat samples from which individual bands were excised, bracketed by standards. (B) Labeling pattern for bands which were excised and reamplified and designation of standards for Octopus Spring cyanobacterial populations (P, C1, B, A, and C9) and C. aurantiacus Y-400-fl (Ca). (C) Identity of bands for which sequence information was obtained.

gions sampled. The periodic temperature fluctuations are due to regular surges of near-boiling source water into the spring and effluent channels. The three sample sites span a range of temperatures between 48 and $72^{\circ} \mathrm{C}$ : a low-temperature site $(48$ to $\left.54^{\circ} \mathrm{C}\right)$, a midrange-temperature site $\left(50\right.$ to $\left.65^{\circ} \mathrm{C}\right)$, and a high-temperature site $\left(56\right.$ to $\left.72^{\circ} \mathrm{C}\right)$. Figure $1 \mathrm{~B}$ shows the DGGE profiles of each triplicate sample taken from within each temperature region. Profiles of samples taken from within the same temperature region are essentially identical, while those from different temperature regions display distinctly different banding patterns. However, some bands appear to be common to different temperature sites.

Figure 2 summarizes the results of DGGE analysis and sequencing attempts for samples from each temperature region. The Octopus Spring standards are shown with the lanes containing PCR amplification products of the different temperature regions. Figure $2 \mathrm{~A}$ shows the original gel from which bands were excised. Figure 2B indicates bands that were excised and for which sequencing was attempted. Some bands within the sample lanes appeared to align with standards, permitting a tentative identification based on their locations in the gradient. For instance, band 4 appears to comigrate with cyanobacterial type B, bands 9 and 12 appear to comigrate with cyanobacterial type A, and bands 5 and 14 appear to comigrate with $C$. aurantiacus.

Before being sequenced, each PCR-reamplified DGGE band was run on a denaturing gradient gel to confirm its position relative to that in the original mat sample (Fig. 3). For example, the PCR products of bands 7 through 9, excised from the 50 to $65^{\circ} \mathrm{C}$ sample, realign with their respective bands in the original sample. The same is true for bands 11 through 13
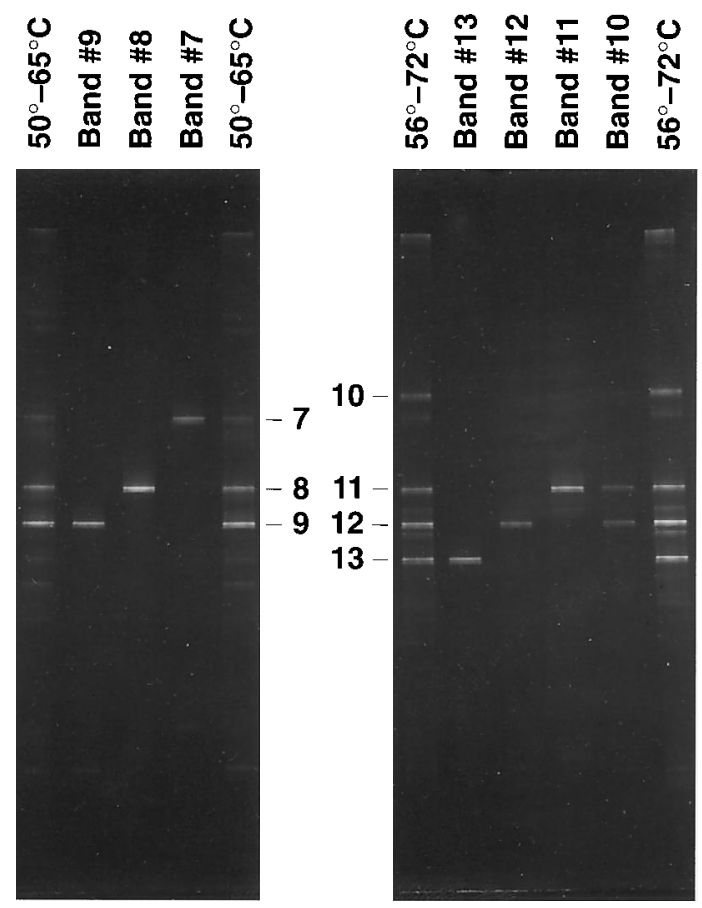

FIG. 3. Representative DGGE gel demonstrating confirmation of band positions of PCR reamplification products from mid- and high-temperature-range Octopus Spring mat samples. Bands are as defined for Fig. 2B. 
A.

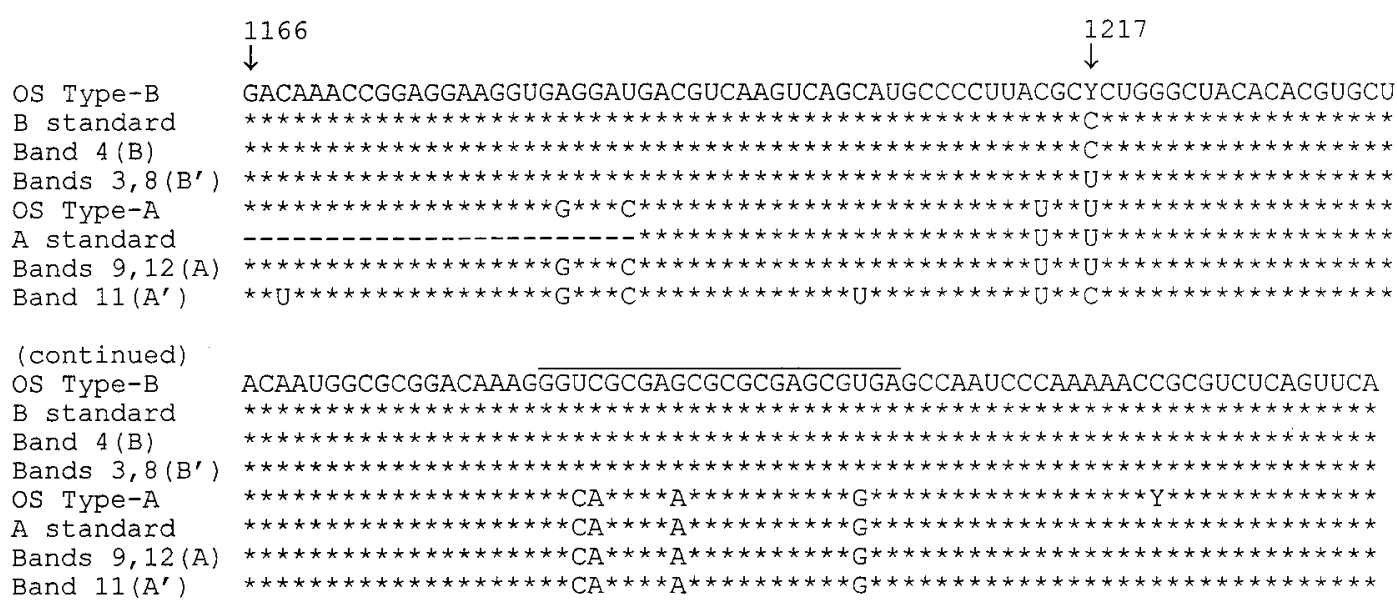

B.

1153

$\downarrow$

Os Type-E CAAGACUGCCUACGCAAgUAgAgAgGAAGGAGgGGAUGACGUCAAGUCCUCAUGGCCCUUNCNCCUAGGGCCACACACGUACUACAA

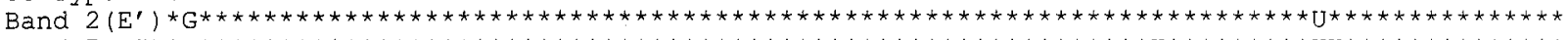

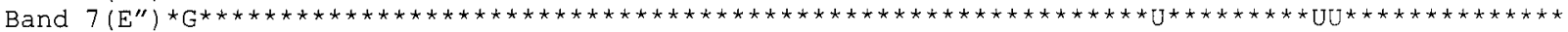

(continued)

OS Type-E UGGGUGCUACAAUGGGCGAAGUCGCGAGACGGAGGUAAUCCCAAAAAAGCACUCUCAGUUCAGAUCGGAGUCUGCAACUCGACUCCG

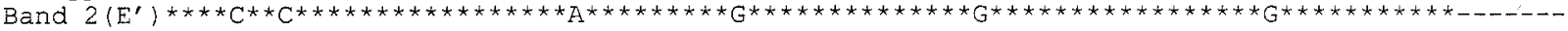

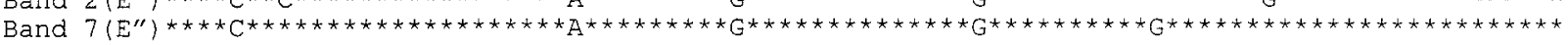

c.

1218

$\downarrow$

OS Type-C UgGgGCGACACACACGCUACAAUgGCGCCGACAAUgCGUCGCUCCCGCGCAAGCGGAUGCYAAUCGCCAAACGGNGCCC

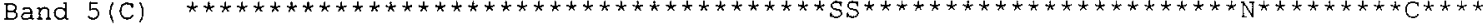

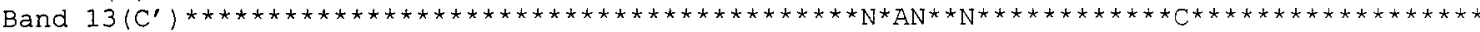

FIG. 4. Comparison of nucleotide differences in DGGE band sequences (designated by band number [sequence type]) with sequences of their closest phylogenetic relatives. (A) Cyanobacterial sequences compared with the Octopus Spring (OS) sequence types A and B. (B) Green sulfur bacterium-like sequences compared with Octopus Spring sequence type E. (C) Green nonsulfur bacterium-like sequences compared with Octopus Spring sequence type C. *, no difference from reference sequence; $\mathrm{N}$, ambiguous nucleotide position. E. coli positions (arrows) and regions to which hybridization probes were targeted (27) (solid overlines) are indicated. The Octopus Spring sequence types are from the Ribosomal Database Project (35).

from the 56 to $72^{\circ} \mathrm{C}$ sample. Band 10 demonstrates the necessity of confirming PCR band reamplifications by DGGE, as the product(s) of the reamplification did not realign with the targeted band. Instead, bands 11 and 12 appear to have been amplified. Clearly, sequencing this product(s) would not yield the sequence of band 10 .

Most excised bands yielded sequence data. Band 14 did not yield sufficient PCR product to attempt sequencing. Band 1 yielded a PCR product, but subsequent sequencing reactions failed to provide usable data. Figure $2 \mathrm{C}$ illustrates bands for which sequence information was obtained. The sequence of each standard was verified. Figure 4A displays differences among cyanobacterial sequence types. The sequence of band 4 is the same as the type B cyanobacterial sequence, confirming the tentative inference based on comigration in the gradient. This is also the case for bands 9 and 12, whose sequences are the same as the type A cyanobacterial sequence. The sequences of bands 3 and 8 are the same and are most similar to type $\mathrm{B}$ but contain at least one nucleotide difference ( $\mathrm{C}$ instead of $\mathrm{U}$ at E. coli position 1217) over the region for which se- quence data were obtained (therefore designated B'). As expected, these bands migrate to the same position in the gradient, slightly above the type B standard (Fig. 2C). The sequence of band 11 is most similar to the type A cyanobacterial sequence but differs in at least three nucleotide positions in the region examined (hence designated $\mathrm{A}^{\prime}$ ). Band 11 migrates to a position above type $\mathrm{A}$ in the gradient (Fig. 2C). The maximum sequence variation in this set of four cyanobacterial sequences is $6.9 \%$ on the basis of this limited set of sequence data through the V9 region.

Bands 2 and 7 (labeled $E^{\prime}$ and $E^{\prime \prime}$ ) have sequences that are similar to Octopus Spring sequence type E (Fig. 4B), which is most closely related to green sulfur bacteria (31). The maximum variation among these three sequences is $6.3 \%$, on the basis of sequence data through the V9 region.

Band 5 has no detectable sequence differences from Octopus Spring clone type C (Fig. 4C), a phylogenetic relative of green nonsulfur bacteria (35), and comigrates with a type $\mathrm{C}$ standard generated from an Octopus Spring clone (data not shown). Band 13 is most similar to Octopus Spring type $C$ but 
differs in at least one nucleotide position over the region sequenced (hence labeled $\mathrm{C}^{\prime}$ ). The difference between the type $\mathrm{C}$ and $\mathrm{C}^{\prime}$ sequences is $3.7 \%$, on the basis of this very limited set of sequence data through the V9 region. Band 13 migrates to a position above type $\mathrm{C}$ in the gradient.

Band 6 has a sequence that has not been previously detected and has no close relative in either the Octopus Spring database or the Ribosomal Database Project. Thus, we designate it a new Octopus Spring sequence type, type Q. Because of the limited sequence data, mainly from a variable region, its phylogenetic affiliation cannot be determined with certainty.

\section{DISCUSSION}

The detection of known Octopus Spring sequence types (cyanobacterial types $\mathrm{A}$ and $\mathrm{B}$ and the green nonsulfur bacterium-like type $\mathrm{C}$ ), previously detected in cloning libraries, by DGGE verifies that this method can be used to study known populations. More importantly, the DGGE method allowed us to discover populations of cyanobacteria that could not be confidently identified by cloning. As illustrated in Fig. 4a, the consensus sequence for type B contains a $\mathrm{Y}$ at $E$. coli nucleotide position 1217 , indicating the presence of either a $U$ or a C. Originally, several B-type clones were sequenced from Octopus Spring libraries, and only minor differences such as this were observed $(32,35,36)$. We could not discern whether the difference was real or possibly due to a polymerase error during type $\mathrm{B}$ cloning or sequencing. By taking a conservative approach, a single type B population was defined (32). With DGGE, there is little doubt that these are two separate type B-like 16S rRNA populations. This could result from two different rRNA operons within a single-organism population (21). The best evidence we have that bands B and B' represent populations of different organisms is that they occur in different samples $\left(\mathrm{B}^{\prime}\right.$, but not $\mathrm{B}$, is observed in the 50 to $65^{\circ} \mathrm{C}$ sample). Since we are amplifying $16 \mathrm{~S}$ rRNA genes, two operons in the same organism population should show the same distribution. Several other new populations were also discovered in this single DGGE gel. These include $\mathrm{A}^{\prime}$, closely related to the type A cyanobacterial sequence; $\mathrm{E}^{\prime}$ and $\mathrm{E}^{\prime \prime}$, both closely related to the type $\mathrm{E}$ green sulfur bacterium-like sequence; and $\mathrm{C}^{\prime}$, most closely related to the type $\mathrm{C}$ green nonsulfur bacterium-like sequence.

DGGE, like cloning and sequencing (31), detects the occurrence of sets of phylogenetically related populations as a consistent pattern of community structure. In the case of cyanobacterial populations, which are both phylogenetically and physiologically related, we suggested that evolutionary specialization (e.g., temperature adaptation [see below]) may have led to the guild structure observed in the mat community (9). The similar patterns of occurrence of green sulfur bacterium-like and green nonsulfur bacterium-like populations suggest that other guilds composed of related populations also occur in the mat. Sets of phylogenetically related populations have also been observed in 16S rRNA studies of several other habitats $(3,4,6,10,11,14,29)$ and might reflect the generality of such an ecological basis for the evolution of microbial biodiversity.

Other populations previously detected by cloning and cultivation were not detected in these samples. For example, no major bands corresponding to type $\mathrm{P}, \mathrm{C} 1$, or $\mathrm{C} 9$ cyanobacterial standards appear in any of the samples. It seems especially surprising that types P and C9 were not detected, since Synechococcus spp. of these types have been cultivated from extincting-dilution enrichments of similar samples (9). This incongruity may simply be a true reflection of the populations present at these sites when the samples were collected. We know little about the distributions of populations within the mat in space and time. It may be that nonrandom environmental changes, such as seasonal fluctuations in photoperiod or light intensity, influence population restructuring within the mat. For instance, samples taken at different times of year may be dominated by different populations. In this regard, DGGE samples were collected in August 1994, whereas samples for cultivation were collected in June 1992. Alternatively, population variation could be driven by stochastic factors, such as disturbance and subsequent succession. This might explain smaller-scale distribution differences. For example, in cultivation experiments, independent samples taken within close proximity yielded different cyanobacterial isolates at extincting dilutions (9).

It is also possible that during the PCR there is some bias against the sequence types of the populations that were not observed $(8,26)$. The primers were designed to recover all $16 \mathrm{~S}$ rRNA sequences of domain Bacteria and did in fact recover sequences from members of three kingdoms in this domain (cyanobacteria and green sulfur-like and green nonsulfur-like bacteria). We have additional evidence that the primers also amplify proteobacterial, gram-positive, and Thermus $16 \mathrm{~S}$ rRNA genes (28). However, we cannot be certain that the primers would detect all populations of domain Bacteria without any bias $(8,26)$. Another possible methodological complication is that in some cases we are comparing populations detected by cDNA synthesis from 16S rRNA (e.g., cyanobacterial populations $\mathrm{J}$, I, and $\mathrm{P}$ ) with populations detected by PCR amplification of 16S rRNA genes.

The primary reason that spatial and temporal heterogeneity of bacterial populations in the Octopus Spring mat has not been thoroughly investigated is the labor-intensiveness of cloning and sequencing, oligonucleotide probing, and cultivation approaches. As a result, current information comes from an extremely limited sample set. We hope that DGGE will provide a rapid approach to screening larger numbers of samples, enabling better resolution of the spatiotemporal distributions of inhabitants of the mat community. DGGE allowed a rapid assessment of the amplifiable sequence types within the Octopus Spring mat community. As seen in Fig. 1, profiles within each temperature region are similar and reproducible, suggesting low population variability. In contrast, differences in profiles between the sites suggest that temperature influences community structure. These results are consistent with the existence of temperature-adapted strains of thermophilic Synechococcus spp. (24) and with hybridization probe experiments that indicated that the type B cyanobacterial population was adapted to lower temperatures than the type A population (27). As seen in Fig. 2C, the DGGE results confirm this pattern of distribution. The type $\mathrm{B}$ sequence is present in the lowtemperature $\left(48\right.$ to $\left.54^{\circ} \mathrm{C}\right)$ sample but is absent from the midrange $\left(50\right.$ to $\left.65^{\circ} \mathrm{C}\right)$ and high-temperature $\left(56\right.$ to $\left.72^{\circ} \mathrm{C}\right)$ samples. The type A sequence is present in the mid- and high-range samples but is absent from the low-temperature sample. However, the DGGE results extend the probe data by allowing a finer resolution between cyanobacterial populations. The probes used in the distribution analysis were designed to be specific for the type A and B cyanobacterial sequences (27). As seen in Fig. 4A, they also complement both the type $\mathrm{A}^{\prime}$ and $\mathrm{B}^{\prime}$ sequences and would have cross-reacted with them. Thus, the order of incidence in cyanobacterial populations from low to high temperature is actually $\mathrm{B}, \mathrm{B}^{\prime}, \mathrm{A}, \mathrm{A}^{\prime}$. If these populations are adapted to different temperatures (as suggested by probe results [27]), this is a particularly intriguing result, given the phylogenetic similarity among these four cyanobacterial populations. It suggests that specialization across a 
temperature range of ca. $20^{\circ} \mathrm{C}$ can be accomplished by relatively little genetic change, thus supporting the temperature strain concept of Peary and Castenholz (24). The degree of difference among the four sequences that we detected (6.9\%) was based on a variable region and overestimates the overall difference, which is more likely to be ca. $3 \%(32,35)$. Other cyanobacterial sequence types within the Octopus Spring mat are much more distantly related and may represent populations adapted to other environmental parameters (9).

We observed some gel-to-gel variations in exact band positions, but relative positions remained stable. A more standardized method of gel preparation should eliminate this variation and allow direct profile comparisons between different gels. We found that it is essential to initially verify the identity of a DGGE band by sequencing, since some sequence types that are known to be quite different migrate to the same positions in the gradient. For example, the C. aurantiacus Y-400-fl band migrates to the same position as the type $\mathrm{C}$ sequence (band 5 in Fig. 2C). Therefore, until the sequence of a band is verified, the position in the gradient alone is not a reliable means of identification. Narrower gradients, longer gels, or PCR amplification of a different region of the 16S rRNA gene may provide a mechanism with which to alleviate this problem.

As with all methods used to study microorganisms in nature, DGGE is not without limitations. Obviously, there are many possible reasons why all populations might not be detected (30), and limited sequence data do not permit a robust evaluation of phylogenetic relationships of the sequence types detected. Still, DGGE offers a rapid means of detecting predominant populations which are PCR amplifiable. Because DGGE bands are sequenced directly, cloning, which may cause bias (25), is eliminated. Furthermore, as separation of different sequences is achieved by electrophoresis, laborious screening necessitated by redundancy of clones in a library is also eliminated. Thus, the increase in the number of samples that can be evaluated and the ability to simultaneously detect many populations enable a more aggressive pursuit of the ecology of native bacteria.

\section{ACKNOWLEDGMENTS}

We thank the National Park Service at Yellowstone for their cooperation and assistance and Mike Madigan for providing C. aurantiacus Y-400-fl

This research was supported by a grant from the National Science Foundation (BSR-9209677).

\section{REFERENCES}

1. Abrams, E. S., and V. P. Stanton. 1992. Use of denaturing gradient gel electrophoresis to study conformational transitions in nucleic acids. Methods Enzymol. 212:71-104.

2. Amann, R. I., W. Ludwig, and K. Schleifer. 1995. Phylogenetic identification and in situ detection of individual microbial cells without cultivation. Microbiol. Rev. 59:143-169.

3. Barns, S. M., R. E. Fundyga, M. W. Jeffries, and N. R. Pace. 1994. Remarkable Archaeal diversity detected in a Yellowstone National Park hot spring environment. Proc. Natl. Acad. Sci. USA 91:1609-1613.

4. Choi, B. K., B. J. Paster, F. E. Dewhirst, and U. B. Gobel. 1994. Diversity of cultivable and uncultivable oral spirochetes from a patient with severe destructive periodontitis. Infect. Immun. 62:1889-1895.

5. DeLong, E. F. 1992. Archaea in coastal marine environments. Proc. Natl. Acad. Sci. USA 89:5685-5689.

6. DeLong, E. F., T. M. Schmidt, and N. R. Pace. 1989. Analysis of single cells and oligotrophic picoplankton populations using 16S rRNA sequences, $\mathrm{p}$. 697-701. In T. Hattori, Y. Ishida, Y. Maruyama, R. Y. Morita, and A. Uchida (ed.), Recent advances in microbial ecology. Japan Science Society Press, Tokyo.

7. Don, R. H., P. T. Cox, B. Wainright, K. Baker, and J. S. Mattick. 1991. "Touchdown" PCR to circumvent spurious priming during gene amplification. Nucleic Acids Res. 19:4008.
8. Farrelly, V., F. A. Rainey, and E. Stackebrandt. 1995. Effect of genome size and $r r n$ gene copy number on PCR amplification of $16 \mathrm{~S}$ rRNA genes from a mixture of bacterial species. Appl. Environ. Microbiol. 61:2798-2801.

9. Ferris, M. J., A. L. Ruff-Roberts, E. D. Kopczynski, M. M. Bateson, and D. M. Ward. Enrichment culture and microscopy conceal remarkable diversity among thermophilic Synechococcus in a single hot spring mat habitat. Submitted for publication.

10. Fuhrman, J. A., K. McCallum, and A. A. Davis. 1993. Phylogenetic diversity of subsurface marine microbial communities from the Atlantic and Pacific Oceans. Appl. Environ. Microbiol. 59:1294-1302.

11. Giovannoni, S. J., T. B. Britschgi, C. L. Moyer, and K. G. Field. 1990. Genetic diversity in Sargasso Sea bacterioplankton. Nature (London) 345: 60-63.

12. Gray, M. W., D. Sankoff, and R. J. Cedergren. 1984. On the evolutionary descent of organisms and organelles: a global phylogeny based on a highly conserved structural core in small subunit RNA. Nucleic Acids Res. 12:58375852 .

13. Kopczynski, E. D., M. M. Bateson, and D. M. Ward. 1994. Recognition of chimeric small-subunit ribosomal DNAs composed of genes from uncultivated microorganisms. Appl. Environ. Microbiol. 60:746-748.

14. Liesack, W., and E. Stackebrandt. 1992. Occurrence of novel groups of the domain Bacteria as revealed by analysis of genetic material isolated from an Australian terrestrial environment. J. Bacteriol. 174:5072-5078.

15. Maniatis, T., E. F. Fritsch, and J. Sambrook. 1982. Molecular cloning: a laboratory manual. Cold Spring Harbor Laboratory, Cold Spring Harbor, N.Y.

16. Muyzer, G., and E. C. de Waal. 1994. Determination of the genetic diversity of microbial communities using DGGE analysis of PCR-amplified 16S rDNA, p. 207-214. In L. J. Stal and P. Caumette (ed.), Microbial mats: structure, development and environmental significance. Springer-Verlag, Heidelberg, Germany.

17. Muyzer, G., E. C. De Waal, and A. G. Uitterlinden. 1993. Profiling of complex microbial populations by denaturing gradient gel electrophoresis analysis of polymerase chain reaction-amplified genes coding for 16S rRNA. Appl. Environ. Microbiol. 59:695-700.

18. Muyzer, G., S. Hottentrager, A. Teske, and C. Wawer. Denaturing gradient gel electrophoresis of PCR-amplified 16S rDNA. A new molecular approach to analyze the genetic diversity of mixed microbial communities. In A. D. I. Akkermans, J. D. van Elsas, and F. J. de Bruijn (ed.), Molecular microbial ecology manual, in press. Kluwer, Dordrecht, The Netherlands.

19. Muyzer, G., A. Teske, C. O. Wirsen, and H. W. Jannasch. 1995. Phylogenetic relationships of Thiomicrospira species and their identification in deep-sea hydrothermal vent samples by denaturing gradient gel electrophoresis of $16 \mathrm{~S}$ rDNA fragments. Arch. Microbiol. 164:165-172.

20. Myers, R. M., T. Maniatis, and L. S. Lerman. 1987. Detection and localization of single base changes by denaturing gradient gel electrophoresis. Methods Enzymol. 155:501-527.

21. Mylvaganam, S., and P. P. Dennis. 1992. Sequence heterogeneity between the two genes encoding 16S rRNA from the halophilic Archaebacterium Haloarcula marismortuii. Genetics 130:399-410.

22. Olsen, G. J., D. J. Lane, S. J. Giovannoni, and N. R. Pace. 1986. Microbial ecology and evolution: a ribosomal RNA approach. Annu. Rev. Microbiol. 40:337-365.

23. Pace, N. R., D. A. Stahl, D. J. Lane, and G. J. Olsen. 1986. The analysis of natural microbial populations by ribosomal RNA sequences. Adv. Microb. Ecol. 9:1-55.

24. Peary, J. A., and R. W. Castenholz. 1964. Temperature strains of a thermophilic blue-green alga. Nature (London) 202:720-721.

25. Rainey, F. A., N. Ward, L. I. Sly, and E. Stackebrandt. 1994. Dependence of the taxonomic composition of clone libraries for PCR-amplified naturally occurring $16 \mathrm{~S}$ rDNA on the primer pair and the cloning system used. Experientia 50:789-801.

26. Reysenbach, A. L., L. J. Giver, G. S. Wickham, and N. R. Pace. 1992 Differential amplification of rRNA genes by polymerase chain reaction. Appl. Environ. Microbiol. 58:3417-3418.

27. Ruff-Roberts, A. L., G. J. Kuenen, and D. M. Ward. 1994. Distribution of cultivated and uncultivated cyanobacteria and Chloroflexus-like bacteria in hot spring microbial mats. Appl. Environ. Microbiol. 60:697-704.

28. Santegoeds, C. M., S. C. Nold, and D. M. Ward. Personal communication.

29. Schmidt, T. M., E. F. DeLong, and N. R. Pace. 1991. Analysis of marine picoplankton community by $16 \mathrm{~S}$ rRNA gene cloning and sequencing. J. Bacteriol. 173:4371-4378.

30. Ward, D. M., M. M. Bateson, R. Weller, and A. L. Ruff-Roberts. 1992 Ribosomal RNA analysis of microorganisms as they occur in nature, p. 219-286. In K. C. Marshal (ed.), Advances in microbial ecology. Plenum Press, New York.

31. Ward, D. M., M. J. Ferris, S. C. Nold, M. M. Bateson, E. D. Kopczynski, and A. L. Ruff-Roberts. 1994. Species diversity in hot spring microbial mats as revealed by both molecular and enrichment culture approaches-relationship between biodiversity and community structure, p. 33-44. In L. J. Stal and P. Caumette (ed.), Microbial mats: structure, development and environmental significance. Springer-Verlag, Heidelberg, Germany. 
32. Ward, D. M., R. Weller, and M. M. Bateson. 1990. 16S rRNA sequences reveal numerous uncultured microorganisms in a natural community. Nature (London) 345:63-65.

33. Ward, D. M., R. Weller, and M. M. Bateson. 1990. 16S rRNA sequences reveal uncultured inhabitants of a well-studied thermal community. FEMS Microbiol. Rev, 75:105-116.

34. Wawer, C., and G. Muyzer. 1995. Genetic diversity of Desulfovibrio spp. in environmental samples analyzed by denaturing gradient gel electrophoresis of
(NiFe) hydrogenase gene fragments. Appl. Environ. Microbiol. 61:2203-2210. 35. Weller, R., M. M. Bateson, B. K. Heimbuch, E. D. Kopczynski, and D. M. Ward. 1992. Uncultivated cyanobacteria, Chloroflexus-like inhabitants, and spirochete-like inhabitants of a hot spring microbial mat. Appl. Environ. Microbiol. 58:3964-3969.

36. Weller, R., J. W. Weller, and D. M. Ward. 1991. 16S rRNA sequences of uncultivated hot spring cyanobacterial mat inhabitants retrieved as randomly primed cDNA. Appl. Environ. Microbiol. 57:1146-1151. 